\title{
FUNCIONES DE PESO
}

\author{
Nancy Moya Lázaro**
}

Resumen: En este artículo, presentamos un estudio analítico de una clase de pesos, damos algunas propiedades y formulamos algunas estimaciones de estos.

Palabras clave: Funciones peso, Espacios de Sobolev con peso

\section{WEIGHT FUNCTION}

\begin{abstract}
In this paper we show an analytic study of a weight class, give some properties and we formulate some estimations of this.
\end{abstract}

Key words: Weight function, Weighted Sobolev spaces

\section{Introducción}

En este artículo hacemos un estudio analítico de una clase de pesos y damos algunas propiedades de los mismos. Presentamos ejemplos importantes de pesos e introducimos una amplia clase de pesos $R_{\rho_{1}, \ldots, \rho_{k}}$ y formulamos algunas estimaciones de estos.

En la Sección $\S 2$, definimos la clase de funciones peso $R_{\rho_{1}, \ldots, \rho_{k}}$, para algunas constantes $\rho_{1}, \ldots, \rho_{k}$ positivas, los cuales son definidos como pesos que pertenecen a $C^{k}\left(\mathbb{R}^{N},(0, \infty)\right)$ y para $x \in \mathbb{R}^{N}$, $|\alpha|=r$, con $1 \leq r \leq k$, satisfacen la siguiente desigualdad

$$
\left|D^{\alpha} \rho(x)\right| \leq \rho_{r} \rho(x), x \in \mathbb{R}^{N} .
$$

Probamos que si $\rho$ está en la clase $R_{\rho_{1}, \rho_{2}}$ entonces su reescalamiento definido por $\rho_{\epsilon}(x):=\rho(\epsilon x)$, también está en la clase $R_{\epsilon \rho_{1}, \epsilon^{2} \rho_{2}}$. Asimismo, los pesos de esta clase verifican estimaciones del tipo $\rho(x) \leq \rho(x-y) e^{\sqrt{N} \rho_{1}|y|}, x, y \in \mathbb{R}^{N}$. En particular, si $x=y$, tenemos que $\rho(x) \leq e^{C|x|} \rho(0)$, donde $C=\sqrt{N} \rho_{1}$. Esto significa que los pesos de la clase $R_{\rho_{1}, \rho_{2}}$ tienen a lo más un crecimiento exponencial en el infinito.

\section{Funciones Peso}

Una función $\rho: \mathbb{R}^{N} \rightarrow(0, \infty)$ continua y estrictamente positiva sera denominada función peso.

Definición 2.1. Diremos que una función peso $\rho: \mathbb{R}^{N} \rightarrow(0, \infty)$ es una función de la clase $R_{\rho_{1}, \ldots, \rho_{k}}$, con $k \in \mathbb{N}$ si:

(i) $\rho \in C^{(k)}\left(\mathbb{R}^{N}\right)$

(ii) $\left|D^{\alpha} \rho(x)\right| \leq \rho_{r} \rho(x)$, para $x \in \mathbb{R}^{N},|\alpha|=r$, con $1 \leq r \leq k$, y para ciertas constantes positivas $\rho_{r}$.

En particular, se dirá que $\rho$ está en la clase $R_{\infty}$ si se verifica la Definición 2.1 para todo $k \in \mathbb{N}$.

\footnotetext{
${ }^{* *}$ UNMSM, Facultad de Ciencias Matemáticas, e-mail: nanesp@yahoo.com
} 
Lema 2.1. Sea $\rho$ un peso de la clase $R_{\rho_{1}, \rho_{2}}$. Entonces se tiene:

(i) $\rho_{\epsilon}(x):=\rho(\epsilon x)$ es un peso de la clase $R_{\epsilon \rho_{1}, \epsilon^{2} \rho_{2}}$.

(ii) $\rho(x) \leq \rho(x-y) e^{\sqrt{N} \rho_{1}|y|}, x, y \in \mathbb{R}^{N}$.

Demostración. (i)es inmediato.

(ii) Observemos que si $\rho \in R_{\rho_{1}, \rho_{2}}$, para cada $x, y \in \mathbb{R}^{N}, t>0$, se tiene:

$$
\frac{|\nabla \rho(x-y t)|}{\rho(x-y t)} \leq \sqrt{N} \rho_{1}
$$

Por otra parte, para $x, y \in \mathbb{R}^{N}, t>0, z=x-y t$

$$
\left|\partial_{t}[\ln \rho(x-y+y t)]\right|=\frac{\left|\partial_{z} \rho(z) \cdot y\right|}{|\rho(x-y+y t)|}=\frac{|\nabla \rho(x-y+y t)||y|}{\rho(x-y+y t)} \leq \sqrt{N} \rho_{1}|y| .
$$

Una simple integración con respecto de $t$ de 0 a 1 en la desigualdad anterior implica la estimación,

$$
\rho(x) \leq \rho(x-y) e^{\sqrt{N} \rho_{1}|y|}, x, y \in \mathbb{R}^{N} .
$$

Observación 2.1. En particular si $x=y$, tenemos que $\rho(x) \leq e^{C|x|} \rho(0)$, donde $C=\sqrt{N} \rho_{1}$. Es decir los pesos de la clase $R_{\rho_{1}, \rho_{2}}$ tienen a lo más un crecimiento exponencial en el infinito.

\section{Ejemplos importantes}

Ejemplo 2.1. i) El peso $\rho(x):=\left(1+|x|^{2}\right)^{\gamma}, \quad x \in \mathbb{R}^{N}, \gamma \in \mathbb{R}$, pertenece a la clase $R=R_{\rho_{1}, \rho_{2}}$ $\operatorname{con} \rho_{1}=2|\gamma|, \rho_{2}=4|\gamma||\gamma-1|+2|\gamma| \cdot \square$

ii) Sea $\rho(x)$ un peso de $C^{2}\left(\mathbb{R}^{N}\right)$ tal que $\rho(x)=e^{\gamma|x|}, \gamma \in \mathbb{R}$ para todo $|x| \geq 1$ entonces $\rho(x)$ está en la clase $R_{\rho_{1}, \rho_{2}}$, para ciertos $\rho_{1}, \rho_{2}$ que dependen de $\gamma_{\cdot \square}$

Además estos pesos verifican las siguientes desigualdades, que son muy útiles en el estudio de las propiedades de regularización de la solución de la ecuación del calor ver en [1], que formulamos en el siguiente lema

Lema 2.2. (i) Para todo $\rho \in R_{\rho_{1}, \rho_{2}}, \frac{\rho(x)}{\rho(y)} \leq e^{\sqrt{N} \rho_{1}|x-y|}$.

(ii) Si $\rho(x)=\left(1+|x|^{2}\right)^{\gamma}$, con $\gamma \in \mathbb{R}$ tenemos que

$$
\frac{\rho(x)}{\rho(y)} \leq C(\gamma)\left(1+|x-y|^{2}\right)^{|\gamma|}
$$

y la siguiente condición de integrabilidad en $\mathbb{R}^{N}$

$$
\int_{\mathbb{R}^{N}}\left(1+|x|^{2}\right)^{\gamma} d x<\infty \text { si y sólo si } \gamma<\frac{-N}{2} .
$$

(iii) Si $\rho \in C^{2}\left(\mathbb{R}^{N}\right)$, tal que $\rho(x)=e^{\gamma|x|}$ si $|x| \geq 1$, con $\gamma \in \mathbb{R}$, entonces satisface,

$$
\frac{\rho(x)}{\rho(y)} \leq C e^{|\gamma||x-y|} \text { para cada } x, y \in \mathbb{R}^{N}
$$

$y$ es integrable si $\gamma<0$. 
Demostración. (i) Es consecuencia inmediata del Lema 2.1 (ii).

(ii) Probar (2.1) es equivalente a probar que $\rho(x) \rho(z) \leq C \rho(x+z)$, para cada $x, z \in \mathbb{R}^{N}$, si $\gamma<0$ y que $\rho(x) \rho(z) \geq C \rho(x+z)$, para cada $x, z \in \mathbb{R}^{N}$, si $\gamma>0$.

Para ambos casos, por la naturaleza de la función $\rho(x)$ y el signo de $\gamma$, sera suficiente verificar una desigualdad del tipo $\left(1+|x+z|^{2}\right)<C\left(1+|x|^{2}\right)\left(1+|z|^{2}\right)$ para cada $x, z \in \mathbb{R}^{N}$. En efecto, por la desigualdad de Cauchy-Schwartz

$$
1+|x+z|^{2} \leq 2\left(1+|x|^{2}\right)\left(1+|z|^{2}\right) .
$$

De (2.3), si $\gamma<0$ se tiene $\left[\left(1+|x|^{2}\right)\left(1+|z|^{2}\right)\right]^{\gamma} \leq\left(\frac{1}{2}\right)^{\gamma}\left(1+|x+z|^{2}\right)^{\gamma}$ y si $\gamma>0$ entonces se tiene $\left(1+|x+z|^{2}\right)^{\gamma} \leq 2^{\gamma}\left[\left(1+|x|^{2}\right)\left(1+|z|^{2}\right)\right]^{\gamma}$. De ambos casos se sigue la parte (ii) del Lema. La condición (2.2) se deduce del uso de coordenadas polares.

(iii) Es una consecuencia de la estructura de $\rho$ y del hecho que es de clase $C^{2}\left(\mathbb{R}^{N}\right)$. En la prueba, consideramos cuatro casos, si $x, y \in B(0,1)$; para, $|x|>1,|y|>1$, en el caso que $|x|>1$ e $y \in B(0,1)$, y finalmente si $x \in B(0,1)$ e $|y|>1$.

Ahora, presentamos el siguiente lema, que será útil en el estudio de las relaciones entre los espacios de Sobolev con peso y sin peso.

Lema 2.3. Si $\rho \in R=R_{\rho_{1}, \rho_{2}, \ldots, \rho_{n}}$ entonces

$$
\left|D^{\alpha} \rho^{w}(x)\right| \leq C \rho^{w}(x), \text { para } \operatorname{cada}|\alpha| \leq n, w \in \mathbb{R} .
$$

donde $C=C\left(n, w, \rho_{1}, \rho_{2}, \ldots, \rho_{n}\right)$. En particular, $\forall w \in \mathbb{R}, \rho^{w} \in R_{\hat{\rho}_{1}, \ldots, \hat{\rho}_{n}}$ con $\hat{\rho}_{1}=\ldots=\hat{\rho}_{n}=C, y$ si $w \in(0,1)$ entonces $\hat{\rho}_{i}$ se puede tomar independiente de $w$.

La prueba la hacemos por inducción sobre el orden.

\section{REFERENCIAS BIBLIOGRÁFICAS}

[1] Comportamiento Asintótico de Soluciones de Ecuaciones de Evolución Ph.D. Thesis, Universidad Complutense de Madrid. (2004). 